\title{
Love apps: cambios y continuidades en el imaginario de las relaciones de pareja
}

\author{
Gabriel Alfonso Corral Velázquez,Jocelyne Alvarez Espinoza ${ }^{1}$
}

\begin{abstract}
Resumen
En los últimos veinte años se han visto una serie de cambios en el modo en que se relacionan los seres humanos. La emergencia de las redes sociodigitales ha supuesto una transformación en el ser y estar dentro del ecosistema mediático; al mismo tiempo que se intercambian mensajes, se pueden compartir fotografías, videos y construir información de manera inmediata. Dentro de este cúmulo de fenómeno se encuentran las aplicaciones para citas: love apps. Dichas aplicaciones forman que parte de las redes sociodigitales no sólo se utilizan como un espacio para generar conversación o mensajería instantánea, sino que buscan facilitar la comunicación entre personas que desean obtener encuentros personales o relaciones de pareja. En este documento se plantea si las love apps han generado cambios o si prevalecen continuidades en el imaginario de las relaciones de pareja, a partir de la experiencia de los usuarios. Por medio de un cuestionario desarrollado y aplicado de manera no probabilística, los usuarios dan cuenta de lo que implica el uso de estas aplicaciones y si consideran ha cambiado el imaginario social de las relaciones de pareja.

\section{Abstract}

The last twenty years have been a changes in our way of relating as human beings. The emergence of social media has meant a transformation in our being within the media ecosystem; at the same time that we exchange messages, we can share photos, videos and build information immediately. Within this context are the: love apps. These applications that are part of social media are not only used as a sphere to generate conversation or instant messaging, but also seek to facilitate communication between people who want to obtain a date or relationships. In this paper it is raised whether the love apps have generated changes or continuities prevail in the imaginary of the couple relationships from the experience of the users. From a questionnaire developed in a non-probabilistic way, users give an account of what the use of these applications entails and whether it has changed the social imaginary of couple relationships.
\end{abstract}

\section{Palabras Clave}

Imaginarios, Redes sociodigitales, Aplicaciones de citas, Usuarios

Keywords

Social imaginary, Social media, Love apps, users

1 Universidad Autónoma de Querétaro,

\section{Introducción}

La etapa de revoluciones tecnológicas que se vive actualmente construye discursos y tendencias con características específicas de los espacios digitales. Diversifican las opciones de comunicación y convivencia porque, la propia naturaleza del internet, permite un acceso holístico a distintos modos de ser y estar.
Las redes sociodigitales son un ejemplo de estos nuevos modos de vinculación. A partir del uso generalizado del internet, colaboran como una herramienta que permite construir un perfil e ir generando conexiones y enlaces con otros usuarios. Se van articulando nodos que facilitan la interacción entre personas desde el mundo online. 
Dentro de este fenómeno se encuentran las aplicaciones para citas: love apps ${ }^{1}$. Este tipo de aplicaciones no sólo se utilizan como un espacio para generar conversación, sino que buscan facilitar la comunicación entre personas que desean obtener encuentros, citas o alguna forma de relación interpersonal más allá de la cotidianidad de las redes de interacción digital común. Para Reig y Vílchez (2013), estas redes son el último eslabón digital que ha venido a transformar la dinámica de las relaciones en pareja.

Estas plataformas han ido insertándose en la vida digital de las personas alrededor del mundo. La compañía de análisis App Annie ${ }^{2}$ expone que dentro de los mercados globales en este rubro, dos nombres de aplicaciones para citas dominaron en 2015: Badoo y Tinder $^{3}$. El estudio elaborado por App Annie exhibe que, si bien estas aplicaciones para citas no se encuentran activas en todos los países del mundo, están presentes en cada uno de los continentes. Al final, tienen y persiguen un alcance global.

Las love apps dominan el mercado de manera diferenciada. En Europa, cuatro son los nombres que resaltan: Badoo, Tinder, Frim y Lovoo. En Asia, domina Badoo, Tinder, Momo, Paktor, Frim, YYC y Heartbeep. En América, predominan Tinder y Badoo. En Oceanía Tinder es la aplicación de citas líder.

El portal UNAM Global Noticias ${ }^{4}$, revela el promedio de edades que las utilizan: los jóvenes de entre 26 y 30 años son los que la ocupan mayoritariamente (32.80\%), seguido está el grupo de 31 a 40 años (32.1\%), de 21 a 25 años (31.3\%), de 41 a 50 años, y mayores de 50 años $(9.7 \%)$. De los cuales el $67.5 \%$ son hombres y el $32.5 \%$ mujeres.

En este sentido, las love apps se plantean como un espacio de redefinición del imaginario sobre las relaciones de pareja. El propio funcionamiento de esta red, sus usos, aplicaciones y naturaleza permiten que el usuario viva una experiencia de

\footnotetext{
${ }^{1}$ Se ha recuperado desde el título y a lo largo del trabajo el término love apps para referirse al objeto de análisis debido a que es el nombre comercial con el cual se conoce a estas redes sociodigitales.

${ }^{2}$ Recuperado en Octubre de 2019 de: https://www.bbc.com/news/resources/idt-6ea9c7ed-efef-4bd7-b38a-ebdd21b7d 4be.

${ }^{3}$ Tinder como aplicación de citas se presenta en 2012 desarrollada por la compañía IAC, en 2014 fue nominada App del Año en los Premios Enter.Co, con más de 50 millones de usuarios. En tanto Badoo es la aplicación de citas que nace en 2006 cuenta con aproximadamente 35 millones de usuarios activos.

${ }^{4}$ Recuperado en octubre de 2019 en http://www.unamglobal.unam.mx/
}

"ligue" distinta a la ordinaria. Las representaciones de pareja, que cada usuario observa a partir de su interacción con esta plataforma, son específicas e individuales.

Los imaginarios, de acuerdo con Castoriadis (1998) son:

Una construcción socio histórica que, pese a su carácter imaginado, opera en la realidad ofreciendo tanto oportunidades como restricciones para el accionar de los sujetos no es una ficción ni una falsedad, sino que se trata de una realidad que tiene consecuencias prácticas para la vida cotidiana de las personas. (Castoriadis, 1998)

Los imaginarios no se oponen a lo real, sino que son producidos a través de prácticas y tecnologías que constituyen la forma en que se expimenta el mundo. Pintos (2005) también expone que los imaginarios son "esquemas socialmente construidos que nos permiten percibir, explicar e intervenir en lo que en cada sistema social diferenciado se tenga por realidad" (Pintos 2005:17).

Bajo esta lógica, según las vivencias que cada usuario de Tinder perciba, será capaz de elaborar su propia definición y referente de pareja. Cada experiencia de los internautas va a construir una realidad distinta, o similar, que lo llevará a accionar de manera específica. Las consecuencias prácticas, que se desplieguen del imaginario de las relaciones en pareja, tendrán que ver con el uso que cada persona tenga de esta plataforma, en este sentido se pregunta: ¿cómo se redefine el imaginario de las relaciones en pareja a partir de las interacciones, encuentros o vínculos que se construyen en los espacios de aplicaciones para citas?

\section{Imaginarios y redes sociodigitales}

El concepto que acoraza a este trabajo es el de imaginario. Castoriadis (1998) lo observa como un proceso creador permanente. Un conjunto complejo de construcciones simbólicas que hacen posible las relaciones entre personas, objetos e imágenes, implican modos de pertenencia, normas comunes y aspiraciones.

Castoriadis (1998), expone que el imaginario no es reflejo de ninguna sociedad determinada, ni de ninguna realidad natural o social, sino que es una construcción simbólica que permite instituir, crear y modificar a las sociedades concretas, a la vez que cada sociedad concreta constituye como imaginario un cúmulo de significaciones específicas. 
Este argumento sirve para ilustrar el objeto de estudio que se desarrolla. El imaginario, que cada persona tiene sobre lo que significan las relaciones de pareja, se forma a partir de construcciones simbólicas que adoptan desde la familia, la escuela, los amigos, la calle, así como en lo que ven y lo que escuchan.

Las sociedades son historia, y suponen tanto formas específicas de auto-alteración, como su propio modo de cambiar y autogenerarse y reconstruirse como un lenguaje, reglas de reproducción de las instituciones, reglas de lo permitido y lo prohibido, de lo lícito y lo ilícito, de las maneras de producir y reproducir la vida material (Castoriadis, 1985:331; Castoriadis, 2002, 2004).

Partiendo de esa premisa, el imaginario que se tiene sobre las relaciones de pareja viene dado por la temporalidad de cada sociedad. La manera en la que se conciben están determinadas por características específicas acordes al espacio, lugar y tiempo. "Los imaginarios asumen cierta historicidad que, al ser reconocidos en tanto construcciones socioculturales, no pueden resistir las reconstrucciones del devenir histórico" (Martínez y Muñoz, 2008, pp. 212).

Desde esta lógica, se sostiene lo expuesto anteriormente: el imaginario es parte de estereotipos -en este caso, el estereotipo occidental que se vive desde hace años y que permea en la concepción de relaciones de pareja-. Asimismo, el argumento de que elegir pareja viene enlazado a un amor romántico exclusivo.

Bernal, Carvajal, Encina y Fernández (2015) plantean que en esta decisión actúan las ideas preconcebidas asociadas al imaginario social que cada quien posee acerca de lo que debe ser el amor y cómo debe ser la pareja que se elija. Las nociones de cómo debe ser esta persona y cómo debe ser la relación que se entable, se componen de diversas influencias que provienen tanto del núcleo familiar como de la sociedad misma.

La búsqueda de pareja basada en un ideal es una práctica de carácter personal, pero también de carácter social, donde intervienen múltiples variables. Según Cuevas (2013), además de las familias; el Estado, los medios de comunicación y las religiones a través de las iglesias, forman ideas sobre el amor que luego se ven materializadas en manifestaciones afectivas, durante toda la vida en pareja.
Estas instituciones promueven ideales del amor y del estar en pareja, que son tomados por los individuos como patrones de comportamiento orientado a la búsqueda y mantención de una relación amorosa. Estos ideales pueden verse materializados en forma de expectativas que son moldeadas de acuerdo con el modelo cultural del que se forme parte. (Bernal, Carvajal, Encina y Fernández, 2015: 7)

En este sentido se afirma que el imaginario que se tiene sobre las relaciones de pareja se ve influenciado por las experiencias de las que se han sido parte. De las tradiciones, mitos y leyendas que, a lo largo de la vida, se ha escuchado sobre lo que significa tener una pareja. Son imaginarios que, si bien son personales, también tienen una fuerte carga cultural, familiar y social.

Entonces, recuperando la idea expuesta por Castoriadis (1985, 2002, 2004), se plantea que el imaginario de las relaciones de pareja supone una creación figurada a partir de las instituciones de la sociedad que se mantiene y damos continuidad; no obstante, la interacción con estas aplicaciones puede en un momento determinado contrastar el imaginario social interiorizado desde hace algunas generaciones.

Por ello se considera pertinente hacer el lazo entre redes sociodigitales, relaciones de pareja y los imaginarios, toda vez que con "la aparición de redes de interacción en plataformas digitales como Facebook, Twitter, WhatsApp, Snapchat, Instagram, Tinder, entre otras, significa una nueva forma de conexión, comunicación y relación con los otros" (Urueña, Ferrari, Blanco y Valdecasa, 2011). Las transformaciones en los vínculos afectan la construcción, el imaginario y el mantenimiento cotidiano de las relaciones de pareja.

Asimismo, Paredes (2012) menciona que las relaciones de pareja, desde el auge de las redes sociales, se han visto afectadas y modificadas por la forma en que la gente las utiliza y el sentido con el cual son llevadas, contrastando desde este punto de vista el imaginario mencionado anteriormente. Se puede afirmar entonces que las redes sociodigitales cumplen hoy un papel importante en la esfera de la vida en pareja. De una forma $\mathrm{u}$ otra interviene, convive y moldea la manera en que las personas están experimentando su situación en pareja. Estos cambios suceden por el uso y ajustes que cada individuo configura en ellas, dependiendo de los fines y objetivos que desea obtener. 
Dicho de otra manera, las redes sociales digitales han cambiado la forma de actuar, de comportarse y regula la manera de compartir e involucrarse como pareja, no sólo en la que se da de manera física o presencial entre la pareja, sino las reglas o el compromiso que se adquiere al momento de llevar o buscar una relación desde el plano virtual. Garibay (2013) plantea que el sistema relacional está en constante cambio y por instinto se dan nuevas tendencias al crecimiento mutuo, de esta manera el sistema relacional de la pareja funciona de forma conjunta con relación a la interacción que se presenta con las redes sociales. Es decir, ambos conceptos están siendo modificados por la propia naturaleza de la temporalidad y, con ello, se van adaptando a la par uno con el otro.

La sociedad y la configuración que de esta se desarrolla en los espacios de interacción virtual enfrenta nuevas necesidades reflejadas en las redes sociales, para saciar carencias comunicativas y de distancia como una forma de adaptación humana. Tal como lo menciona Gainza (2003), esta sociedad tiene transformaciones desde dos dimensiones: la modernidad y las tecnologías en un medio de globalización y en ella se encuentran las relaciones de pareja.

\section{Love apps y los usuarios}

Entre otras cosas, por ejemplo, el rechazo en la app no es comparable con el rechazo físico, probablemente por la impersonalidad o el anonimato. Es un híbrido perfecto. Esta modalidad contribuye a que el usuario se sienta en mayor confianza de exponer cuáles son sus ambiciones en la plataforma. Lo peor que le puede suceder, es no obtener el famoso match..$^{5}$

Las love apps comparten funcionalidades con las redes sociales en tanto que los usuarios tienen que construir un perfil público en un sistema delimitado, articulan una lista de usuarios con los que se establecen vínculos y generan, a su vez, relaciones con los amigos de otros amigos.

Esta aplicación puede concebirse como un espacio en donde los parámetros del tipo de relación que los usuarios buscan se definen desde un inicio. Con su descripción, el internauta tiene la libertad de filtrar sus términos y restricciones. Condiciona a la otra persona, antes de conocerse,

\footnotetext{
${ }^{5}$ Hacer match refiere al emparejamiento surgido por Tinder cuando la validación de dos usuarios es recíproca.
}

a que acepte, rechace o se adapte al tipo de vínculo relacional que desea obtener.

En esta red de aplicaciones, ya no se trata solamente de ser candidato para obtener un encuentro con alguien, sino de tener la posibilidad de diseñarlo. Por ello, estas plataformas ofrecen al usuario diversos filtros que puede ir seleccionando de acuerdo con sus gustos y preferencias. Se van conformando cadenas que prometen al usuario ubicar al tipo de pareja que desean encontrar.

“El fenómeno del ligue en internet incluso catapultó a Tinder, Badoo, Happn, Bumble y Grindr como las segundas más buscadas/utilizadas sólo detrás de Twitter e Instagram en México", expone la consultora The Competitive Intelligence Unit. ${ }^{6}$.

\section{Las love apps son:}

"La promesa de estas apps es poder encontrar potenciales candidatos para una relación sin depender de la presencia física, haciendo más accesible y controlable el encuentro con un otro. Las love apps pueden interpretarse como una de las expresiones de un ideal romántico cuyos valores progresivamente se asemejan con la dinámica y la lógica del mercado del deseo" (Pavoni 2017, p. 7).

Se plantea la creencia de que estas redes, aunque prometen acercamientos románticos y afectivos, también han generado un fin de deseo sexual. Los usuarios ven en Tinder la posibilidad de redefinir sus relaciones de pareja; de crear acuerdos que beneficien a las dos partes; de intercambiar placeres y pasiones efímeras. La propia love app Tinder señala en su portal de internet que "con 30 mil millones de matches hasta la fecha, Tinder® es la aplicación más popular para conocer gente. Piensa en nosotros como tu acompañante de confianza, vayas donde vayas, allí estaremos. nos llaman "la app más hot del mundo. ${ }^{\text {es }}$ por algo: conseguimos encender la llama entre personas con más de 26 millones de matches cada día". 7

Por tal, se argumenta que la dinámica flexible de las love apps ha modificado la percepción y el imaginario que se tiene sobre las relaciones en pareja y las dinámicas vinculadas con ellas.

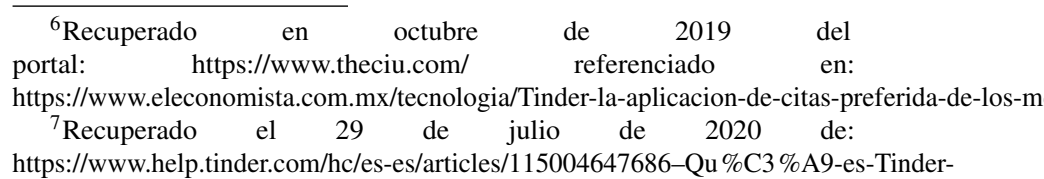




\section{Perspectiva de análisis}

Se ha elegido como ejemplo de análisis la aplicación para citas Tinder porque, según Fernández (2017), es la app que ha revolucionado la forma en la que la gente se conoce en todo el mundo. Tinder es una aplicación que permite a los usuarios ponerse en contacto con otras personas según criterios como distancia física, género, preferencia sexual, edad, entre otros.

Las dos características distintivas de Tinder son el swipe y el match. La primera de ellas consiste en la posibilidad de pasar de un usuario a otro, al descartarlo o aceptarlo, con un sencillo movimiento del dedo; mientras que el match es el emparejamiento surgido por Tinder cuando la validación de dos usuarios es recíproca. En este sentido, y con este mecanismo, Pavoni (2017) explica que Tinder facilita el acceso y representan un ejemplo concreto del proceso de tecnificación que se ha desatado sobre las relaciones afectivas más íntimas.

Se ha convertido en una vía de acceso para comprender las expectativas y estrategias que los sujetos modernos despliegan para establecer relaciones afectivas, depositando en ese proceso algo tan importante como su deseo de estar acompañados en una multiplicidad de formas posibles. Pareciera que el uso, interacción, encuentro y vínculos que surgen en esta aplicación para citas, construye, modifica o cambia la perspectiva e imaginario que los individuos tienen alrededor de lo que significan las relaciones de pareja.

Con base en este planteamiento, se va a trabajar con jóvenes y adultos de ambos sexos. La muestra de 30 usuarios activos de las redes sociodigitales, seleccionados de manera aleatoria a través de una convocatoria abierta en redes. Se parte del supuesto de que existe un imaginario de relaciones de pareja que se está redefiniendo a partir de las interacciones, encuentros o vínculos que se construyen en los espacios de aplicaciones para citas: Love Apps. Por lo tanto, el objetivo es identificar los imaginarios de pareja que tienen los usuarios de aplicaciones para citas y observar las tendencias de los imaginarios de pareja, a partir del uso de las aplicaciones para citas.

Como afirma Bisquerra (2004), al estudio de los significados de las acciones humanas, así como de la vida social y del cambio, Chárriez (2012) añade que este modelo trata de interpretar cómo se crea la estructura básica de la experiencia, sus significados, su continuidad y su participación a través del lenguaje y de otras construcciones simbólicas.

A los participantes se les aplicó un cuestionario conformado por 11 preguntas abiertas en las que el objetivo fue obtener un panorama general sobre los usos y perspectivas que han tenido estas personas con las aplicaciones para citas (en caso de que las hayan utilizado alguna vez). Se buscó aprovechar las distintas vivencias que cada participante ha tenido en la plataforma, de esta manera, se buscó analizar la definición del imaginario de cada participante sobre las relaciones de pareja a partir de los espacios de aplicaciones para citas, específicamente en Tinder. Es decir, se analizó el referente del mundo social del que han sido parte, en sus propias palabras, contemplar.

Las variables del cuestionario estuvieron conformadas de la siguiente manera.

A través de un formulario de Google, se realizó un sondeo con las preguntas expuestas anteriormente. En total, se obtuvieron 30 respuestas. A continuación se expone la discusión sobre los resultados obtenidos.

Los 30 cuestionarios se dividen de la siguiente manera: siete personas tienen 22 años; cinco tienen 24 años; y dos de 21, 25, 2737 y 52 años respectivamente. Una persona de $19,23,31,34,39$ y 51 años respectivamente .13 son del género femenino y 17 del masculino. 14 de los encuestados respondieron que son estudiantes; tres son docentes; dos profesionales independientes; dos ingenieros; dos trabajan y estudian; y las siguientes ocupaciones corresponden una a cada encuestado restante: periodista, mercadólogo, operador, gerente, encargado de ventas, electricista y empleados. 25 de los encuestados respondieron que son solteros; cuatro son casados y una persona clasifica su relación como noviazgo.

Figura 1. ¿Qué es una relación de pareja?

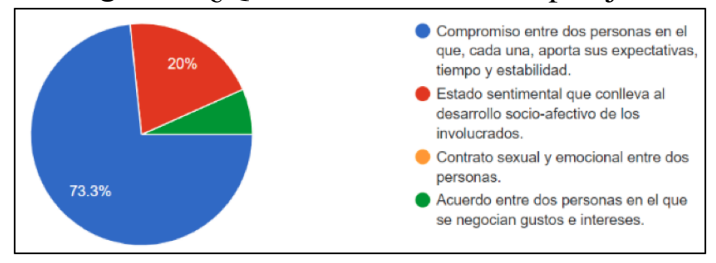

El grueso de los participantes en el cuestionario coincide en que una relación de pareja es una asociación de dos seres humanos compuesta por un sentimiento amoroso, en donde 
Tabla 1. Operacionalización del estudio de los imaginarios

\begin{tabular}{|c|c|c|}
\hline Imaginario & $\begin{array}{l}\text { Construcción } \\
\text { simbólica y } \\
\text { cultural que } \\
\text { las personas } \\
\text { elaboran a } \\
\text { partir de las } \\
\text { experiencias y } \\
\text { vivencias de } \\
\text { las que han } \\
\text { sido parte. }\end{array}$ & $\begin{array}{l}\text { 1) Culturalmente, } \\
\text { cómo ha sido pensado } \\
\text { su imaginario de } \\
\text { pareja desde la } \\
\text { familia, los amigos, } \\
\text { la escuela, la calle. } \\
\text { 2) De estos } \\
\text { estereotipos } \\
\text { culturales, cuáles ha } \\
\text { adoptado al momento } \\
\text { de construir su propio } \\
\text { imaginario de pareja. } \\
\text { 3) En términos } \\
\text { prácticos, cómo } \\
\text { ha vivido este } \\
\text { imaginario en sus } \\
\text { relaciones de pareja. } \\
\text { 4) Este imaginario de } \\
\text { pareja ha cambiado a } \\
\text { partir de trasladarlo } \\
\text { a términos de } \\
\text { plataformas digitales. }\end{array}$ \\
\hline $\begin{array}{l}\text { Relación de } \\
\text { pareja }\end{array}$ & $\begin{array}{l}\text { Acuerdo entre } \\
\text { dos personas } \\
\text { en el que, } \\
\text { cada una, } \\
\text { aporta sus } \\
\text { expectativas, } \\
\text { compromiso } \\
\text { y estabilidad } \\
\text { que conllevan } \\
\text { al desarrollo, } \\
\text { o no, } \\
\text { socioafectivo } \\
\text { del individuo. }\end{array}$ & $\begin{array}{l}\text { 5) En términos } \\
\text { generales, pero } \\
\text { concretos, cómo han } \\
\text { sido sus relaciones de } \\
\text { pareja. } \\
\text { 6) Estas experiencias, } \\
\text { tienen cercanía con } \\
\text { la construcción de } \\
\text { relación de pareja que } \\
\text { usted ha creado. } \\
\text { 7) Sus relaciones de } \\
\text { pareja son diferentes } \\
\text { cuando se consolidan } \\
\text { desde el ámbito } \\
\text { digital. } \\
\text { 8) Para ellos, qué } \\
\text { es una relación de } \\
\text { pareja. }\end{array}$ \\
\hline $\begin{array}{l}\text { Redes } \\
\text { sociodigitales }\end{array}$ & $\begin{array}{l}\text { Espacios de } \\
\text { comunidades } \\
\text { virtuales } \\
\text { en las que } \\
\text { individuos, } \\
\text { con } \\
\text { expectativas } \\
\text { similares, se } \\
\text { relacionan, } \\
\text { comunican } \\
\text { y comparten } \\
\text { gustos e } \\
\text { intereses. }\end{array}$ & $\begin{array}{l}\text { 9) Si han utilizado } \\
\text { alguna vez } \\
\text { plataformas digitales } \\
\text { para conseguir pareja. } \\
\text { 10) } \mathrm{Su} \text { experiencia } \\
\text { en estas plataformas } \\
\text { cumplió con sus } \\
\text { expectativas iniciales } \\
\text { 11) Su idea de } \\
\text { relación de pareja } \\
\text { cambió al momento } \\
\text { de vivirla desde una } \\
\text { esfera digital. }\end{array}$ \\
\hline
\end{tabular}

cada uno aporta expectativas sucesivas, duración y estabilidad que conllevan al desarrollo socio afectivo del individuo. En 28 respuestas se sostiene que una relación de pareja no es ni un contrato ni un acuerdo, es un compromiso formal $\mathrm{y}$ socioafectivo. Por otro lado, dos respuestas consideran que una relación de pareja es un acuerdo entre dos personas en el que se negocian gustos e intereses. Ninguno de los participantes eligió la respuesta de "contrato sexual y emocional entre dos personas".

Figura 2. ¿Qué ámbito ha influenciado más en tu construcción de lo que significa una relación de pareja?

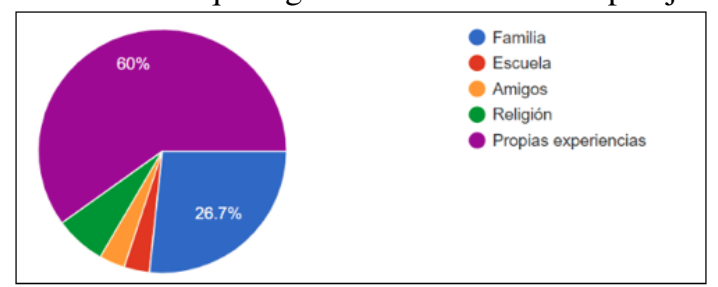

Los imaginarios se forman a partir de construcciones simbólicas y culturales. Dichas construcciones se pueden adoptar desde la familia, la religión, la escuela, los amigos, la calle, lo que cada persona ve y escucha. También se consideran que las nociones de cómo son las relaciones de pareja se componen de diversas influencias que provienen tanto del núcleo familiar como de la sociedad misma. En 18 respuestas se afirma que lo que más ha influenciado en su construcción de lo que significa una relación de pareja son sus propias experiencias. Ellos han creado el imaginario desde las raíces de sus vivencias porque han sido personales y puestas en práctica. Ocho respuestas coinciden que su definición de relación de pareja viene desde la influencia familiar. Dos consideran que la religión ha sido su fuente de referencia. Finalmente, una persona respondió que sus amigos han influenciado más y otro contestó que ha sido la escuela.

Figura 3. ¿Cuál de estos estereotipos has adoptado al momento de elaborar tu propia definición de relación de pareja?

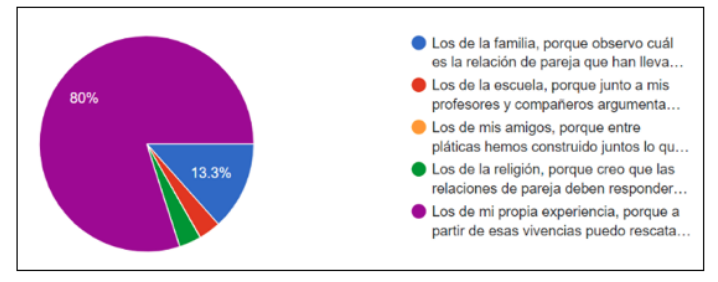


24 personas respondieron que de los estereotipos mencionados, obtuvieron el suyo al considerar que la relación de pareja se sostiene desde una cuestión cultural y simbólica. Por otro lado, cuatro respuestas consideran que estos estereotipos los han adoptado desde la familia porque se han guiado por la relación de pareja que tienen sus padres. Ninguno respondió que ha sido influenciado por sus amigos. En estas líneas es posible observar que los imaginarios de relaciones de parejas son construidos desde terrenos culturales, simbólicos, sociales y colectivos.

Figura 4. En términos generales, ¿cómo calificas tus relaciones de pareja?

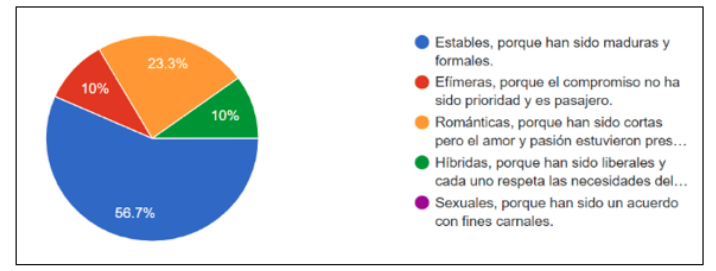

Se considera que una relación da origen a esto siempre y cuando haya un mantenimiento a través del tiempo donde se presente duración y estabilidad. En estos términos, en 17 cuestionarios se encontraron respuestas que sostienen que sus relaciones de pareja han sido estables porque fueron maduras y formales. Siete mencionaron que sus relaciones han sido románticas porque son cortas pero el amor y pasión están presentes. Tres más contestaron que sus relaciones han sido efímeras porque el compromiso no ha sido prioridad y es pasajero; mientras que tres respondieron que sus relaciones han sido híbridas porque son liberales y cada uno respeta las necesidades del otro.

Figura 5. Estas experiencias, ¿tienen cercanía con el significado de relación de pareja que usted ha construido?

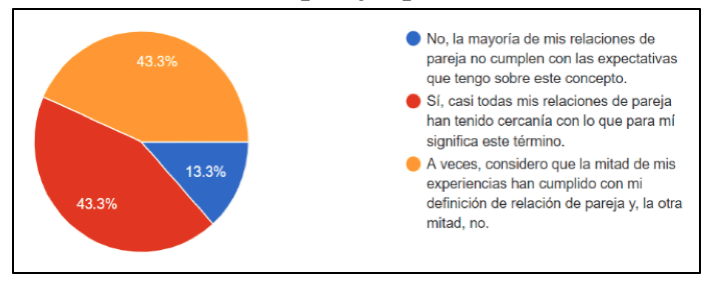

Las sus experiencias sobre las relaciones de pareja sí han tenido cercanía con el significado que han construido sobre este término a partir de las referencias socioculturales que constituyen los imaginarios.
Figura 6. ¿Alguna vez has utilizado aplicaciones para citas?

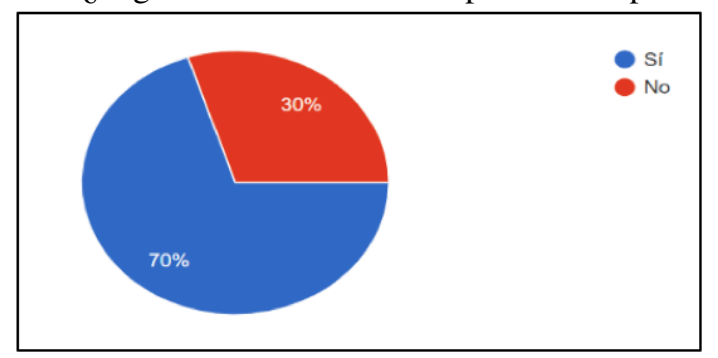

El 70 por ciento de los participantes respondieron que sí han utilizado alguna vez aplicaciones para citas, en tanto que el otro 30 por ciento contestaron que nunca lo han hecho.

Figura 7. ¿Has conseguido una relación de pareja desde estas plataformas?

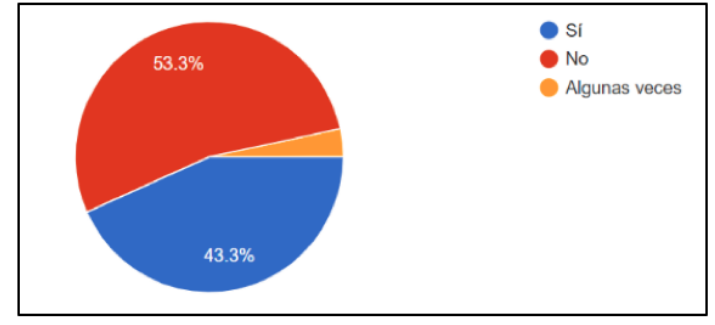

Más de la mitad de las respuestas refieren a que en ninguna oportunidad en las love apps han conseguido una relación de pareja desde estas plataformas de citas. El $43 \%$ contestó que sí, al menos una vez una relación de pareja desde estas aplicaciones y una respondió que sólo algunas veces.

Figura 8. En general, ¿cómo describe estas relaciones de pareja?

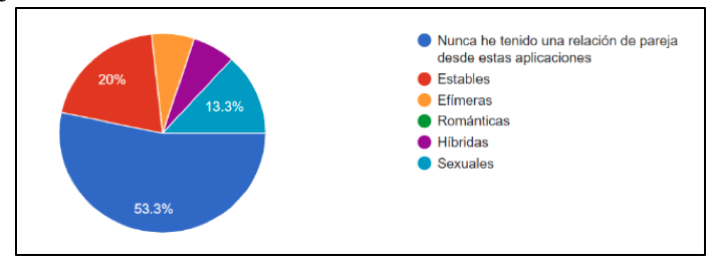

Es significativa esta respuesta en conjunto con el de la pregunta anterior, puesto que se puede relacionar que en al menos 16 personas prefieren consolidar sus relaciones desde la dinámica tradicional cara a cara, fuera de los terrenos digitales. Por otro lado, en seis casos respondieron que, en general, sus relaciones de pareja desde las aplicaciones para citas han sido estables. Cuatro contestaron que han sido sexuales, dos efímeras y dos híbridas. 
Figura 9. ¿Ha cambiado tu idea sobre la relación de pareja a partir de vivirla desde una esfera digital?

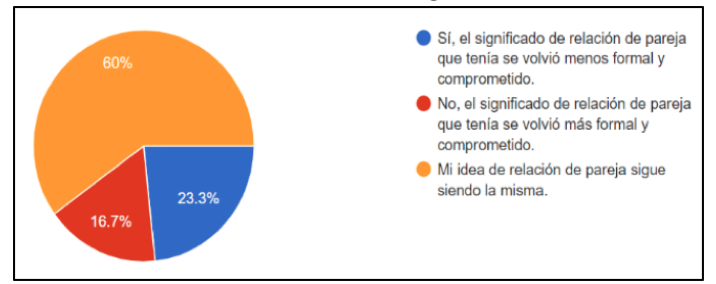

El imaginario de las relaciones de pareja que se tiene no se bifurca y persiste cuando se traslada a terrenos digitales (específicamente desde las plataformas de aplicaciones para citas) ya que el $60 \%$ de las respuestas mantiene la idea que sobre estas tiene. Por otro lado, siete respuestas mencionaron que sí cambió su idea de relación de pareja al vivirla desde una esfera digital porque se volvió menos formal y comprometida. Por último, 12 respuestas coinciden en que la dinámica de las aplicaciones para citas inevitablemente modifica la percepción y el significado que se tiene sobre las relaciones en pareja.

La mayoría, sin embargo, no vieron alterada su percepción sobre las relaciones de pareja. Esto da muestra de la complejidad implicada en la construcción de imaginarios. Este término refiere procesos de larga duración en los que se entremezcla información y percepciones de distintas fuentes con variados grados de credibilidad. No se modifican por una experiencia sino por las distintas interpretaciones que se pueden dar en virtud de lo que pueden encontrar en el uso de la aplicación.

Figura 10. ¿Consideras que las relaciones de pareja son distintas cuando se consolidan desde las aplicaciones para citas?

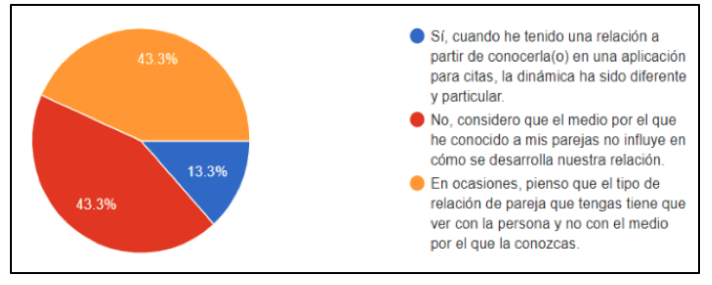

Poco más del $43 \%$ de las respuestas consideran que el tipo de relación de pareja tiene que ver con la persona y no con el medio por el que la conozca; otro $43 \%$ que contestó que sus relaciones de pareja nunca han sido distintas cuando se consolidan desde los terrenos de las aplicaciones para citas. Piensan que el medio por el que han conocido a sus parejas no influye en cómo se desarrolla su relación. Mientras que el $13 \%$ de las personas respondieron que sus relaciones de pareja sí son distintas cuando se construyen desde los espacios de las aplicaciones para citas porque la dinámica ha sido diferente y particular. De lo anterior se rescata que, la mayoría de las experiencias de los encuestados no coincide con lo que exponen algunos autores quienes plantean que los cambios en las relaciones de pareja, desde el auge de las redes sociales, se han visto afectadas y modificadas por la forma en que la gente las utiliza y el sentido en el cual son llevadas. Las experiencias digitales de los encuestados tienen cercanía con sus vivencias offline.

Figura 11. ¿Cuál ha sido la razón principal por la que has utilizado las aplicaciones para citas?

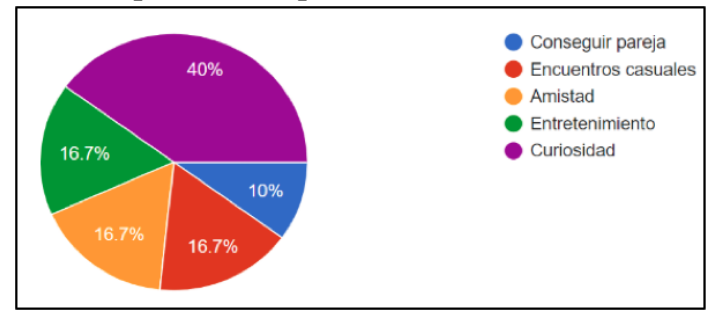

El $40 \%$ de las respuestas coinciden que la principal razón por la que han utilizado aplicaciones para citas es por curiosidad. Fragmentado, el 16\% contestaron que sólo por entretenimiento; por encontrar amistades y en busca de encuentros casuales. Sólo el 10\% mencionó que su principal razón ha sido conseguir pareja. Si bien, las redes sociales cumplen hoy un papel importante en la vida en pareja al intervenir y moldear dinámicas de búsqueda de pareja, estos cambios no suceden por la propia naturaleza de las redes sociales, sino por el uso y ajustes que cada individuo configura en ellas, dependiendo de los fines y objetivos que desea obtener.

\section{Discusión y cierre}

Una relación de pareja es una asociación de dos seres humanos compuesta por un sentimiento amoroso, en donde cada uno aporta expectativas sucesivas, duración y estabilidad que conllevan al desarrollo socioafectivo del individuo. Bajo esta lógica, 28 de las 30 respuestas coinciden con la referencia. Para estas personas una relación de pareja no es ni un contrato ni un acuerdo, es un compromiso formal, estable y socioafectivo. En este sentido, se preguntó sobre el entorno que más ha influenciado en la construcción del 
significado de una relación de pareja. Para comparar las respuestas de los participantes, se tomó como referencia lo que plantea Castoriadis (2004), sobre los imaginarios y en este caso concreto el imaginario de las relaciones de pareja; la experiencia personal más el entorno sociocultural en el que se han desenvuelto la persona ha sido lo que ha construido el imaginario de las relaciones de pareja.

Al mismo tiempo, resulta interesante que en el imaginario hay expectativas que se reproducen y lo fortalecen: confianza recíproca, respeto, afecto, diálogo y ayuda mutua, generando paulatinamente vínculos de apego. Es importante señalar que puede ser diferente el significado de relación de pareja que una persona pueda tener por cómo las ha vivido y experimentado. El imaginario en ocasiones es distinto en la práctica. De lo anterior se puede observar que, en la mayoría de los encuestados, el imaginario que han construido sobre las relaciones de pareja no se queda aislado en el pensamiento, sino que tiene relación y coherencia en la práctica. Coincide en ambos terrenos.

En términos de redes sociodigitales la mayoría de las respuestas coinciden en el uso de las love apps, no obstante, las personas prefieren consolidar sus relaciones desde la dinámica tradicional, off line, fuera de los terrenos digitales. En función de lo anterior, los usuarios muestran interés y curiosidad sobre el uso de las love apps, más por curiosidad que por cambios en los imaginarios, puesto que aún existe arraigo en las prácticas que suponen las ideas tradicionales sobre las relaciones de pareja. En su mayoría, las experiencias de relaciones de pareja de quienes participaron en el cuestionario sí tienen cercanía con sus dinámicas fuera del entorno digital. Sus vivencias desde las aplicaciones para citas son similares a las del ámbito fuera de él.

Lo expuesto en este cuestionario, y con los resultados obtenidos, puede permitir el planteamiento de preguntas sobre cómo estamos involucrando las dinámicas on line y off line aún después de tantos años de interacción entre ambas. Las redes sociodigitales, en concreto las love apps, están presentes en nuestro ecosistema mediático. No obstante para el caso que nos ocupa, aún requerimos por nuestra naturaleza, quizá, de la interacción off line para dar significado al intercambio simbólico que supone una relación interpersonal. En tal sentido, los imaginarios construidos y apropiados en la sociedad sobre las relaciones de pareja no han desaparecido, permanecen, y se fortalecen con todo lo que suponen las love apps.

\section{Referencias}

Bernal, P., Carvajal, B., Encina, K. y Fernández, A. (2015). Jóvenes y uso de Facebook: implicaciones en el imaginario de amor y en la proyección de un ideal de pareja. Pontificia Universidad Católica de Valparaíso. Viña del Mar.

Bisquerra Alzina, R. (2004). Metodología de la investigación educativa. Madrid: La Muralla.

Castoriadis C. (1998). Hecho y por hacer. Pensar la imaginación. Edudeba, Buenos Aires.

Castoriadis C. (2002). Figuras de lo pensable. México D.F.: Fondo de Cultura Económica.

Castoriadis C. (2004). Sujeto y verdad en el mundo histórico-social. Buenos Aires: Fondo de Cultura Económica.

Chárriez, M. (2012). Historias de vida, una metodología de investigación cualitativa. Universidad de Puerto Rico.

Cuevas, A. (2013). Contexto familiar y elección de pareja: una aproximación a través de madres solas. Estudios Sociológicos XXXI.

Domínguez, L., Forero, N., y Buitrago, V. (2018). Redes sociales y relaciones de pareja: una aproximación al vínculo desde la interacción cotidiana. Villavicencio.

Fernández, D. (2017). Entendiendo relaciones de pareja en la era Tinder: análisis cualitativo y propuestas educativas. Universidad de Oviedo.

Malagon, P., Cuestas, J. y Reyes, I. (2014). Influencia del Facebook en el mantenimiento de las relaciones de pareja en estudiantes de la Universidad Piloto de Colombia. Bogotá D.C.

Martínez, J. y Muñoz, D. (2008). Aproximación teórico-metodológica al imaginario social y las representaciones colectivas: apuntes para una comprensión sociológica de la imagen. Colombia: Universidad de Manizales.

Miranda, E. (2014). El imaginario social bajo la perspectiva de Cornelius Castoriadis y su proyección en las representaciones culturales de Cartagena de Indias. Universidad de Cartagena.

Pavoni, F. (2017). El ideal romántico en las publicaciones de Tinder y Happn. Representaciones sobre la búsqueda de un otro en el mercado del deseo. Ciudad Autónoma de Buenos Aires: Universidad de Buenos Aires.

Penagos. (2006). Apego, relaciones románticas y autoconcepto en adolescentes bogotanos.. Universitas Psychologica. 
Pintos, Juan L. (2005). Inclusión / Exclusión. Los imaginarios Sociales de un Proceso de construcción social. Revista Sémata, Facultad de Geografía e Historia.

Reig, D. y Vílchez, L. (2013). Los jóvenes en la era de la hiperconectividad: tendencias, claves y miradas. Madrid: Fundación Telefónica y Fundación Encuentro.

Urueña, A., Ferrari, A., Blanco, D. y Valdecasa, E. (2011). Las redes sociales en Internet. Observatorio Nacional de las Telecomunicaciones y de la SI. 